\title{
Numeralität - eine unterschätzte Domäne der Grundbildung?
}

\section{Ausgewählte Forschungsbefunde}

\author{
Anke Grotlüschen · Klaus Buddeberg $(\mathbb{D}) \cdot$ Gabriele Kaiser
}

Eingegangen: 26. Juni 2019 / Angenommen: 25. September 2019 / Online publiziert: 16. Oktober 2019

(C) Der/die Autor(en) 2019

Zusammenfassung In diesem Beitrag werden Begriffe und Forschungsstand zur Numeralität Erwachsener systematisierend zusammengestellt. Die Befunde und Theorien sind nach ihrem Bezug zum deutschsprachigen Diskurs der Erwachsenenbildung ausgewählt und zugleich in einen historischen Kontext, aber auch in den internationalen Diskurs eingebettet. Viele jüngere Befunde erweitern den Ansatz von Jean Lave dahingehend, dass sich Numeralität durch eine hohe Relevanz von Faustregeln und Schätzungen sowie durch weniger formale Vorgehensweisen von der Schulmathematik unterscheidet. Numeralität wird deshalb oft nicht als Mathematik verstanden und bleibt eher unsichtbar. Hinter numeralen Praktiken stehen zudem gesellschaftliche Machtverhältnisse. Auch die Durchsetzung eigener Interessen basiert auf Numeralität - etwa bei finanzieller Grundbildung, im Gesundheitssystem oder bei der Nutzung statistischer Daten. Nicht zuletzt gehen numerale Kompetenzen und Praktiken mit besseren Lebensumständen einher.

Schlüsselwörter Numeracy · Grundbildung · New Literacy Studies · Numeracy als soziale Praxis · PIAAC

Prof. Dr. A. Grotlüschen · Dr. K. Buddeberg $(\bowtie) \cdot$ Prof. Dr. G. Kaiser

Universität Hamburg, Hamburg, Deutschland

E-Mail: klaus.buddeberg@uni-hamburg.de

Prof. Dr. A. Grotlüschen

E-Mail: anke.grotlueschen@uni-hamburg.de

Prof. Dr. G. Kaiser

E-Mail: gabriele.kaiser@uni-hamburg.de 


\section{Numeracy - an underestimated domain of adult basic education?}

Selected research findings

Abstract In this article, terms and the state of research on adult numeracy are compiled in a systematic way. The findings and theories are selected according to their relation to the German-language discourse of adult education and at the same time embedded in a historical context as well as in international discourse. Many recent findings confirm Jean Lave's approach in that numeracy differs from school mathematics in the high relevance of rules of thumb and estimates as well as in less formal procedures. Numeracy is therefore often not understood as mathematics and remains rather invisible. Numeracy practices are based on social power relations. The assertion of one's own interests is also based on numeracy-for example in financial literacy, in the health system or in the use of statistical data. Last but not least, numeracy skills and practices go hand in hand with better living conditions.

Keywords Numeracy · Adult basic education - New literacy studies · Numeracy as social practice $\cdot$ PIAAC

\section{Einleitung und Vorgehensweise}

„Numeralität“ wird im Folgenden mit Bezug auf frühe Arbeiten von Jean Lave verstanden als Rückgriff auf Zahlen und Daten im Alltagsleben, wobei auch Schätzungen und Plausibilitätsprüfungen, Vereinfachungen und Faustregeln angewendet werden (Lave 1988). Das so charakterisierte Forschungsgebiet der Numeralität von Erwachsenen ist jedoch bisher noch nicht als Thema der Zeitschrift für Weiterbildungsforschung, der Hessischen Blätter für Volksbildung oder der Zeitschrift weiter bilden hervorgehoben worden. Demgegenüber haben die Forschungsgegenstände Alphabetisierung, Literalität oder Grundbildung Erwachsener an verschiedenen Stellen den Status eines Heftthemas erhalten. ${ }^{1}$

Die seit 2005 im Zuge der BMBF-Förderung ausgebaute Forschung und Praxis in diesem Feld fokussiert ebenfalls weit überwiegend die Literalität, nicht aber auf Numeralität. Im Rahmen dieser Förderlinien finden sich lediglich zwei Praxisprojekte: zum einen das Projekt „Curriculare Vernetzung - Schuldnerberatung als Ausgangspunkt für Grundbildung (CurVe)“ zur finanziellen Grundbildung unter Leitung des Deutschen Instituts für Erwachsenenbildung (Mania und Tröster 2014) sowie das „Rahmencurriculum Schreiben und Rechnen“ des Deutschen Volkshochschulverbands. ${ }^{2}$ Auch die LEO-Studie richtet sich allein auf Literalität, nicht auf Numeralität (Grotlüschen et al. 2019b).

In England und Frankreich - zwei Länder, die für die Entwicklung der deutschen Alphabetisierungsförderung Pate standen - wurde in je zwei bevölkerungsreprä-

\footnotetext{
1 So etwa in REPORT - Zeitschrift für Weiterbildungsforschung (Heft 3/2011 und 1/2012), in Hessische Blätter für Volksbildung (Heft 2/2014) oder in DIE Zeitschrift für Erwachsenenbildung (Heft 1/2009 und 3/2014).

2 https://www.alphadekade.de/de/ein-lehrplan-fuer-die-grundbildung-1833.html.
} 
sentativen Studien die Literalität und Numeralität Erwachsener erhoben (ANLCI - Agence Nationale de la Lutte contre l'Illetrisme 2005; DfES 2003). Die Folgestudien zeigten Stagnation oder leichte Verbesserung im Bereich Literalität, jedoch Verschlechterungen im Bereich Numeralität (Harding 2011; Jonas 2012).

Für Deutschland liegen Daten zur Numeralität Erwachsener seit der PIAACErhebung vor (Rammstedt 2013). Eine Nutzung dieser Daten ist jedoch zumindest in der deutschen Erwachsenenbildung kaum vorhanden und erfolgt am ehesten noch in der österreichischen Erwachsenenbildung (Schlögl 2016).

Darüber hinaus ist in der deutschsprachigen Literalitätsforschung eher ein Bezug zu Theorien aus dem Bereich der New Literacy Studies erkennbar (umfassend hierzu Linde 2008). Dem lassen sich Arbeiten auf Basis hierarchischer Kompetenzmodelle gegenüberstellen (Rammstedt 2013). Beide Theorieansätze sind auch hinsichtlich der Numeralität Erwachsener im internationalen Raum bekannt und erzeugten umfangreiche Forschungsaktivitäten (Evans et al. 2019; Gal et al. 2009; Tsatsaroni und Evans 2014), sie wurden jedoch in Deutschland bisher kaum rezipiert.

Aufgrund dieser Dominanz der Literalitäts- gegenüber der Numeralitätsforschung stellt sich zunächst die Frage, ob Numeralität eine Relevanz für Erwachsene hat, die nicht bereits durch Literalität abgedeckt ist. Literalität scheint unabdingbar zu sein. Aber braucht es Alltagsmathematik im Erwachsenenleben? Zur Klärung dieser Frage wird im Beitrag in einem historischen Exkurs unter anderem auch auf Adam Rieses Rechenbuch sowie auf Fragen der Arbeiterbildung eingegangen.

Weiterhin stellt sich die Frage, welche spezifischen Subpopulationen in besserer oder schlechterer Weise numeralisiert sind und in welcher Weise es Teilhabeeinschränkungen bei geringer Numeralität gibt. Solche Fragen werden typischerweise mit bevölkerungsrepräsentativen Datensätzen bearbeitet. Dem gegenüber sind Fragen der Verschiedenartigkeit numeraler Praktiken besser im Theoriediskurs der Numeracy as Social Practices zu bearbeiten. Dieser begünstigt zudem qualitativ-empirische Vorgehensweisen. Beide Theoriestränge und empirische Herangehensweisen werden zunehmend rezipiert (exemplarisch zu einem Themenheft der Zeitschrift ZDM Mathematics Education, vgl. Askew, 2015). Darüber hinaus sind Arbeiten im Gefolge der PIAAC-Studien und des Ansatzes Numeracy as Social Practice zu nennen.

In Deutschland wurde auf Basis dieser Diskussionen seit 2014 ein Forschungsprojekt, bestehend aus sechs Teilprojekten, vorbereitet. Dieses wurde 2017 durch die Landesforschungsförderung Hamburg bewilligt und wird seitdem durchgeführt. Die sechs Teilprojekte sind personell so ausgestattet, dass eine Reihe qualitativer oder sekundäranalytisch-quantitativer Arbeiten zur Numeralität entstehen. ${ }^{3}$

Die nachstehende Zusammenschau greift nun diejenigen Themen und Forschungsfragen auf, die in den Diskussionsbeiträgen der vergangenen Jahre zur Sprache gebracht wurden. Dabei wird der aus der deutschen Erwachsenenbildung publizierte Forschungsstand zur Numeralität Erwachsener referiert. Eingebettet wird dieser Diskurs in jene zwei Diskursstränge, die ursprünglich die deutsche

\footnotetext{
${ }^{3}$ Zur Verankerung der eigenen Beiträge wurde eine interdisziplinäre Konferenz mit rund siebzig Vorträgen abgehalten (2018).
} 
Literalitätsforschung beeinflussten: einerseits die bevölkerungsrepräsentative Kompetenzforschung und andererseits die New Literacy Studies.

Dabei musste eine Auswahl getroffen werden. Zum einen basieren wir unsere Ausführungen auf Befunden zu Numeracy as Social Practice, die bereits 2018 in einem Sammelband publiziert wurde, der auch Beiträge aus höchst unterschiedlichen Ländern präsentierte (,Numeracy as social practice. Global and local perspectives“"). Hieraus sind jene Beiträge ausgewählt worden, in denen der hegemoniekritische Theorieansatz besonders deutlich hervortritt. Zum anderen werden Passagen referiert, die die Forschungshistorie aus der Perspektive des Numeracy as Social Practice berichten.

Die Auswahl aus Analysen der PIAAC-Studien beginnt zunächst mit den Hauptergebnissen der OECD-Publikationen, die die Relevanz von Numeralität betreffen. Diese Befunde werden dann mit subgruppenspezifischen Differenzierungen aus der Erwachsenenbildungsforschung in Deutschland ergänzt. Befunde aus den internationalen PIAAC-Konferenzen der OECD und den US-amerikanischen PIAAC-Konferenzen wurden rezipiert, aber zurückgestellt. Sie zeigen jedoch, dass Numeralität durchaus Beachtung gefunden hat, inzwischen auch in einem Themenbericht der OECD (Jonas 2018). International wird Numeralität Erwachsener insofern durchaus thematisiert, in Deutschland jedoch bisher eher wenig.

Die Diskussion der finanziellen Grundbildung hat demgegenüber ihren Platz in der deutschsprachigen Erwachsenenbildungsforschung solide etabliert, wie sich in der Publikationsaktivität zeigt. Auch wenn es sich nicht im engsten Sinne um Numeralitätsforschung handelt, sind die Beiträge u.E. zu würdigen, weil sie zu den Wegbereitern der deutschen Numeralitätsforschung zu zählen sind.

Dieser Beitrag hat das Ziel, die Themen der Numeralitätsforschung für ein deutschsprachiges Publikum in der Erwachsenenbildung anhand der oben angedeuteten Diskurse aufzubereiten. Dabei treffen quantitative auf qualitative Ansätze. Er profitiert von Perspektiven der Mathematikdidaktik, der Sozialarbeit und der Erwachsenenbildung.

Wir fragen zunächst nach der Relevanz der Numeralität von Erwachsenen und stellen Numeralität damit in den Rahmen normativer, aufklärerischer bzw. humanistischer Bestrebungen. Dem folgen die begriffliche Einordnung und Abgrenzung von anderen Konzepten. Der anschließende historische Blick zeigt weniger die humanistisch-normativen Bestrebungen, sondern eher die entsprechende Befundlage. Der Diskurs zur Numeracy as Social Practice schließt sich daran an. Die finanzielle Grundbildung, die Anleihen in beiden Diskursen nimmt, wird darauffolgend gewürdigt. Anschließend werden Sekundäranalysen aus der Kompetenzforschung rezipiert. Einen besonderen Raum nimmt die gebündelte Darstellung jüngster, zur Publikation angenommener Ergebnisse aus der deutschen Numeralitätsforschung ein. Da diese Analysen Anlass und Rahmen für das gesamte Heft der ZfW darstellen, nimmt diese Zusammenfassung etwas mehr Raum ein. Es handelt sich um verteilte Publikationen, die bisher noch an keiner Stelle aggregiert wurden. Im Fazit wird auf einen Handlungsbedarf verwiesen: Dieser kann Praxis, Politik oder vertiefte Forschung betreffen. Nicht zuletzt werden Forschungsdesiderate ausgewiesen, insbesondere der Bedarf nach theoretischer Klärung. 


\section{Does Numeracy Matter? Zur Relevanz von Numeralität}

Die Bedeutung von Numeralität wird heute offiziell derjenigen von Literalität gleichgesetzt, so auch in der Dekade für Alphabetisierung und Grundbildung in Deutschland (Bundesministerium für Bildung und Forschung und Kultusministerkonferenz 2016) und in den Sustainable Development Goals (Nachhaltigkeitszielen) der Vereinten Nationen. Diese enthalten einen Bereich für Bildung (Ziel 4). Darin wird auf Literalität und Numeralität der Bevölkerungen weltweit eingegangen (Ziel 4.6). Diese Parallelität der Bedeutung von Literalität und Numeralität ist jedoch weder in der Forschung noch in der Praxis abgebildet, Numeralität Erwachsener galt lange Zeit als nicht hinreichend erforscht und theoretisiert (Coben 2003; Carpentieri et al. 2009). „Much of the research on numeracy is schools-based, the bulk on teaching the individual elements and operations of numeracy. Adult numeracy is a relatively new concern" (NRDC Institute of Education 2010, S. 5). Mittlerweile hat die internationale Numeracy-Forschung jedoch Fahrt aufgenommen (Askew 2015).

Dabei ist der in der Erwachsenenbildung verwendete Begriff „Numeralität“ von in der Schulforschung überwiegend verwendeter ,mathematischer Literalität“ zu unterscheiden. Bei Erwachsenen kommen alltagsnahe Handlungen (Schätzungen, Überschlagsrechnungen oder -messungen) zum Tragen (Lave 1988).

Daran schließt sich die Tradition der Situated Literacies an, auch bezeichnet als Literacy as Social Practice (Grotlüschen et al. 2019c). Und jüngst wurden Theoriebezüge aufgearbeitet und empirische Studien zu Numeracy as Social Practice (Yasukawa et al. 2018) publiziert.

Auf der anderen Seite wurden im Gefolge von Large-Scale-Assessments, insbesondere mit der seit der Mitte der 1990er durchgeführten International Adult Literacy Survey (IALS), Literalität und Numeralität auf die politische Agenda gesetzt. Numeralität ist in vielen Ländern ein dezidierter Teil der Grundbildungs- oder Literalitätsstrategien für Erwachsene (z. B. England, Australien, Frankreich, Deutschland). Im internationalen Diskurs ist Numeralität Erwachsener verschiedentlich aufgearbeitet worden (Coben 2003; Gal et al. 2009; Carpentieri et al. 2009) und häufig an kritisches Denken gekoppelt (Askew 2015; Geiger et al. 2015a, 2015b).

\section{Alphabetisierung, Grundbildung, Alltagsmathematik, Kompetenzen und Praktiken}

\subsection{Von der Alphabetisierung zur Grundbildung}

„Grundbildung“ umfasst Lesen, Schreiben, Rechnen sowie finanzielle, gesundheitliche, digitale und politische Grundbildung (Duncker-Euringer 2017; Tröster 2000) und löst sukzessive den Begriff der auf den Schriftspracherwerb eingegrenzten „Alphabetisierung" ab (Abraham und Linde 2010). Zwar ist 2011 von einer Nationalen Strategie für Alphabetisierung (Kultusministerkonferenz Arbeitskreis Weiterbildung 2015) und seit 2015 von einer Nationalen Dekade für Alphabetisierung die Rede, doch ist darin bereits ein breiter Kanon von Kompetenzdomänen angelegt, der dem Begriff „Grundbildung“ eher entspricht als dem der „Alphabetisierung“. 
In der deutschsprachigen Erwachsenenbildung ist Alltagsmathematik Teil des Kanons der Grundbildung, wie eine qualitativ-empirische Dissertation zum Begriff zeigte (Euringer 2016b, 2016a). Demzufolge wird Alltagsmathematik in vielen Kultusministerien und bei zentralen Akteuren der Dekade für Alphabetisierung einhellig als Bestandteil eines Grundbildungskanons betrachtet.

Zudem existiert eine zur internationalen Literalitätsforschung quer liegende Grundbildungsdefinition (Tenorth 2004). Diese setzt „Grundbildung“ als Voraussetzung für daran anschließende Bildung. Versteht man den Begriff so, dann lässt er sich nicht in Subdomänen unterteilen. Ebendies ist im internationalen Diskurs aber durchgeführt worden und wurde in den deutschen Diskurs eingeführt, z. B. zu Gesundheitsgrundbildung (Schaeffer et al. 2016), politischer Grundbildung (Menke und Riekmann 2017), digitaler Grundbildung (Wolf und Koppel 2017) oder finanzieller Grundbildung (Mania und Tröster 2015). Dass es hier nicht um schlichte Anpassungsgrundbildung gehen kann, wurde an anderer Stelle pointiert angemerkt (Grotlüschen 2018, S. 1269). Lesen und Rechnen, so zeigt der historische Rückblick, wurden dezidiert zum Zwecke der Aufklärung vorangetrieben.

\subsection{Alltagsmathematik als Teil der Grundbildung}

Die Begriffe „Alltagsmathematik“, „Numeracy“ und „Numeralität“ werden in diesem Beitrag synonym verwendet und von ,Schulmathematik“ unterschieden. Alltagsmathematik zeichnet sich gegenüber Schulmathematik dadurch aus, dass genaue Berechnungen sukzessive dem Überschlagen weichen (Lave 1988, 1993) und dass letzteres häufig nicht als Mathematik wahrgenommen wird (NRDC Institute of Education 2010). Laut Coben (2003) und Tout (1997) ist Numeralität jedoch nicht etwa weniger als Mathematik, sondern mehr als das, denn sie enthalte die Nutzung von Mathematik in all ihren Facetten, wie Raum und Zeit, Maße, Daten und Statistiken etc., um damit aus der praktischen Welt Sinn zu generieren: „That is why we can say that numeracy is not less than maths but more. It is why we don't need to call it critical numeracy - being numerate is being critical" (Tout 1997, S. 13).

Das Konzept wurde in der Kompetenzforschung in der OECD-Studie Programme for the International Assessment of Adult Competencies (PIAAC) auf Erwachsene bezogen (mit PIAAC zu verstehen als Personen im Alter von 16 bis 64 Jahren):

In PIAAC bezeichnet (...) alltagsmathematische Kompetenz die Fähigkeit, sich mathematische Informationen und Ideen zugänglich zu machen, diese anzuwenden, zu interpretieren und zu kommunizieren, um so mit mathematischen Anforderungen in unterschiedlichen Alltagssituationen Erwachsener umzugehen (Zabal et al. 2013, S. 47).

Die Definition bezieht sich auf Kompetenzen, die sich in kontextualisierten Praktiken ${ }^{4}$ niederschlagen.

\footnotetext{
4 Als Kontexte werden im Rahmen der PIAAC-Studie angegeben: „Im privaten Alltag, zum Beispiel bei häuslichen Reparaturmaßnahmen oder bei der Bestimmung einer Fahrtroute. Im Beruf, zum Beispiel bei der Erstellung von Budgets oder Kostenvoranschlägen, beim Lesen von Bauplänen oder bei der Durchführung von Messungen. Im gesellschaftlichen Kontext, zum Beispiel um gesellschaftliche Themen anhand
} 


\subsection{Von (numeralen) Kompetenzen zu (numeralen) Praktiken}

Der Paradigmenwechsel von Kompetenzen zu Praktiken ist auch theoretisch mit der Bewegung der New Literacy Studies (Street 2003) breit ausgebaut.

Das Erwachsenenkonzept alltagsmathematischer Praktiken fußt auf ethnologischen Studien, tonangebend entfaltet von Jean Lave, die den Ansatz der Communities of Practice auf Basis westafrikanischer Feldforschung entwickelt hat (Lave und Wenger 1991; Lave 1993). Einer ihrer Schüler, Stephen Reder, konnte in einem US-amerikanischen Längsschnitt mit jungen Erwachsenen zeigen, dass sich literale Kompetenzen mit und ohne Unterricht nur langsam verbessern, während sich literale Praktiken durchaus weiterentwickeln (Reder 2011).

„Kompetenzen“ sind Handlungsdispositionen, die situationsspezifisch zum Einsatz kommen, sie gelten zudem als erwerbbar (Klieme und Hartig 2008). „Praktiken“ sind dem gegenüber situationsspezifisch in Gebrauch genommene Kompetenzen. Eine Praktik ist ohne die Situation nicht verständlich (Barton und Hamilton 2003). ${ }^{5}$

Inzwischen ist die Kompetenzforschung in der PIAAC-Studie um Hintergrundfragen der Kompetenznutzung erweitert worden. Neben dem psychometrischen Test von Numeralität wurde im Hintergrundfragebogen auch nach der Nutzung von numeralen Kompetenzen gefragt (diese werden als „Skill-Use-Fragen“, als „Fragen der Kompetenznutzung“ oder als „,numerale Praktiken“ bezeichnet). Eigenständige empirische Effekte der Kompetenznutzung (unter Kontrolle soziodemografischer Variablen und der getesteten Kompetenzen) kann unter anderem Stephen Reder nachweisen (Grotlüschen et al. 2016).

\section{Gutenberg und Ries: Lesen und Rechnen zur Volksaufklärung}

Lesekompetenz wird von Luthers Bibel-Übersetzung über Gutenbergs Buchdruck bis hin zu Wilhelm von Humboldts sprachorientiertem Bildungsideal als Herzstück der Aufklärung und Emanzipation betrachtet (Conrad et al. 1998), wenngleich Humboldt im Königsberger Lehrplan auch die Rolle der Mathematik betont (Humboldt 1809). Die Geschichte des Lesens Erwachsener ist von den Lesezirkeln des Bürgertums bis zur Arbeiterbewegung breit erforscht (Conrad et al. 1998; Trumann 2009). ${ }^{6}$ So wie Gutenbergs Bibel (1452-54) als Meilenstein der Geschichte der Volksaufklärung verstanden wird, lässt sich Adam Ries’ zweites Rechenbuch (1522) als Meilenstein

\footnotetext{
von Statistiken, etwa Kriminalitäts- und Gesundheitsstatistiken, zu verstehen. Im Kontext von Aus- und Weiterbildung, zum Beispiel für kaufmännische Lehrgänge“ (Zabal et al. 2013, S. 48).

5 So etwa ist das senkrecht untereinander angeordnete Niederschreiben von Lebensmittelbezeichnungen zwar ein Zeichen von Schriftsprachkompetenz, jedoch wird es erst im situationalen Kontext zur Praktik einer Einkaufsliste.

${ }^{6}$ Unter dem Titel ,Volksaufklärung“ wird z. B. aus geschichtswissenschaftlicher Perspektive überwiegend das Lesen beforscht, nicht etwa das Rechnen. Dies ist exemplarisch bei Ernst Hinrichs vorzufinden, der die Lehrpläne im Raum Oldenburg auswertet: Um 1687 wird „,gesungen, gebetet, in der Bibel gelesen, in Büchern und Briefen gelesen, Buße getan, die Lection aufgesagt, hernach wird geschrieben, es folgen Auslegung und Fragestücken“ (Hinrichs 1998, S. 41), allerdings sei 1751 die Aufsagerei einem reellen Schulbetrieb gewichen: „Vor allem aber: lesen, schreiben und - nota bene - rechnen haben sich zu veritablen Unterrichtsstoffen entwickelt“ (ebd., S. 42).
} 
der Geschichte des Rechnens interpretieren. Und dieses stellt Ries durchaus auch in den Dienst der Verbesserung der Lebensumstände der einfachen Bevölkerung. Er begründet sein Buch im Vorwort wie folgt: „Damit der arme gemeine Mann beim Brotkauf nicht übervorteilt werde" (Deschauer 2013, S. 155). Statt der römischen Zahlen wurde so das dezimale System verbreitet, besonders Kaufleute und Handwerk profitierten davon (ebd., S. 2).

Rechnen ist unabdingbar zur Bewältigung des Alltags, allein um Lohnbetrug und Übervorteilung zu verhindern. Die Mathematikdidaktik hat diesen Fragen seit Jahrzehnten Beachtung geschenkt, so wurden Aufsätze im Themenfeld „Lohnerhöhungen in mathematischer Behandlung"von Getrud Effe und anderen (1976) zusammengetragen. Gabriele Kaiser konnte in den 1980er Jahren (Kaiser-Meßmer 1986) aufzeigen, wie Sachrechnen, d. h. angewandtes Rechnen, im Zuge der Industrialisierung eingeführt wurde. Es wurde nötig, dass die Landbevölkerung, die zunehmend in den Fabriken arbeitete bzw. arbeiten musste, mit Größen umgehen konnte, also mit Geld, Gewichts- und Längenmaßen sowie Zeitspannen. Letztere sind besonders wichtig, wie in den Fabrikordnungen deutlich wird, die die Einhaltung des pünktlichen Arbeitsbeginns gegenüber den ehemaligen Landarbeitern, die an naturbedingte Zeitrhythmen gewöhnt waren, mit drakonischen Strafen durchsetzten.

Daneben gibt es die an den Gymnasien praktizierte Anwendungsorientierung. Mit Beginn der zweiten industriellen Revolution, die insbesondere eine Revolution der optischen Industrie war, wurde es nötig, Schüler (es gab damals nur Jungen) soweit auszubilden, dass sie ein Ingenieursstudium aufnehmen konnten (sog. „Meraner Lehrpläne“" von 1904).

Unter dem Titel „Eine objektive, doch gefährliche Rechenstunde“ publizierte Otto Felix Kanitz bereits 1924 aus der Perspektive der Arbeiterbewegung über die Notwendigkeit des Rechnens. Dieser Entwicklungsstrang wurde in der Diskussion der Erwachsenenbildung über Alltagsmathematik bisher nicht zur Kenntnis genommen.

In der Alphabetisierung Erwachsener geht die Theorieentwicklung, zumal unter UNESCO-Einfluss, auf Paulo Freire zurück, der Alphabetisierung in den Dienst der Befreiung von gesellschaftlicher Unterdrückung stellte (Freire 1996). Dabei blieb Alltagsmathematik jedoch unberücksichtigt - zu Unrecht, denn auch Adam Ries sah sein Rechenbuch als Mittel zur Emanzipation von illegitimer Bevormundung (Deschauer 2013). Alltagsmathematik kann insofern als notwendig erachtet werden, um politische Informationen zu verstehen, um in gesellschaftlichen Verteilungskonflikten zu agieren und um informierte Entscheidungen zu treffen (zu den Kontexten vgl. Zabal et al. 2013, S. 48; zum Forschungsstand OECD and PIAAC Numeracy Expert Group 2009, S. 13).

Numeralität ist auch am Arbeitsplatz relevant, wie Yasukawa zeigt. Sie skizziert zunächst zentrale Einsichten der frühen Studien von Baker, Harris, Lave, Evans und Nunes, die im Laufe der 1990er Jahre publiziert wurden (Yasukawa 2018, S. 225). Diese Generation konnte erstens zeigen, dass Mathematik am Arbeitsplatz deutlich anders aussieht als die Art von Mathematik, die in Schulen geübt und wertgeschätzt wird. Zweitens wurde hier bereits gezeigt, dass mathematische Praktiken am Arbeitsplatz aufseiten der Beschäftigten nicht immer als mathematische Aktivitäten wahrgenommen werden. Drittens ist der Transfer von Wissen aus der Schule an den Arbeitsplatz weder geradlinig noch unproblematisch (ebd.). 
Die von Yasukawa weiterhin aufgearbeiteten jüngeren Studien seit den 2000er Jahren bestätigen, dass Vorgesetzte als „Gatekeeper“ fungieren, die Beschäftigten mathematische Informationen vorenthalten und lediglich Umsetzungsinformationen weitergeben. Weiterhin wurden unzutreffende Defizitdiskurse über numerale Praktiken jüngerer Beschäftigter festgestellt. Nicht zuletzt wurde ein dominanter Diskurs überlegener schulischer Mathematik herausgearbeitet, der sich als Gegensatz zu den als inferior angesehenen numeralen Praktiken darstellt. Das wiederum gilt laut Yasukawa als Grund dafür, dass sich erwachsene Beschäftigte nicht als Personen verstehen, die Mathematik anwenden oder betreiben, sondern nur mehr als jemand, der schätzt, überschlägt und überprüft (a.a.O., S. 226).

\section{Numeracy as Social Practice: Kontexte und Machtverhältnisse}

Yasukawa, Rogers, Jackson und Street (Yasukawa et al. 2018) publizierten jüngst eine Aufsatzsammlung, die die Theorieentwicklung zum übergreifenden Konzept Numeracy as Social Practice entfaltet. Die zentrale Annahme lautet, dass Numeralität nicht wert- oder kontextfrei, sondern in Machtverhältnisse eingebettet ist. Sie beziehen sich dabei nicht allein auf Streets Lebenswerk, sondern auch auf drei Generationen der Cultural-Historical Activity Theory (CHAT) seit Vygotsky, Leontiev und Engeström und nicht zuletzt auf Laves frühe Arbeiten zum situierten Lernen.

Die Befunde zeigen zunächst, inwiefern die jeweilige Umgebung mathematischer Aktivitäten die verschiedenen numeralen Praktiken beeinflussen (Rogers und Street 2018). Rogers und Street fassen Einzelstudien über neuseeländischen KiwiObstanbau, mexikanische Landarbeit, Berufsvorbereitung in Schweden sowie Reisanbau auf den Philippinen zusammen. In den Studien wurde herausgearbeitet, dass Schätzungen anstelle von Berechnungen als schneller und daher effizienter wahrgenommen werden. Zugleich bestätigt sich, dass selbst diejenigen, die erfolgreiche Überschlagsrechnungen praktizieren, sich selbst als mathematisch wenig kompetent betrachten (Kane 2018, S. 34).

Mathematik ist dabei nicht frei von Machtverhältnissen, z. B. zwischen Arbeitgebern und Beschäftigten. Beispielsweise erfordert das Abzahlen von Schulden in einem betriebseigenen Geschäft in Mexiko genaue Kontrolle der Lohnnachweise und Schuldscheine, um der Gefahr von Übervorteilung durch den Betrieb zu entgehen (Kalman und Solares 2018). Das zeigt sich auch in Südafrika, wo alle Curricula nach der Apartheid überarbeitet worden, mit Ausnahme der Mathematik, die als neutral angesehen wurde. Ein südafrikanischer Beitrag (Khuzwayo 2018) zeigt mit dem Titel „Occupation of our Minds“, dass bisherige Curricula durch diesen Neutralitätsdiskurs fortgeschrieben werden, obwohl sie im Sinne dieser Theorierichtung nicht als neutral zu verstehen sind. Khuzwayo argumentiert, dass es unter dem ApartheidRegime keineswegs beabsichtigt war, Farbige in Mathematik zu unterrichten. So sollte unterbunden werden, dass die Macht der weißen Minderheit hinterfragt und letztlich Widerstand dagegen aufkommen würde. Dass Mathematik an den sozialen Kontext gebunden ist, zeigt sich auch in Botswana (Nthogo Lekoko et al. 2018). Hier wird Numeralität in von Kursen für Social Empowerment eingesetzt, indem alltägliche numerale Praktiken zur Sprache gebracht werden. Allerdings, so zeigt sich 
in Australien, wird aus verbesserter Anerkennung numeraler Praktiken noch keine Forderung nach Lohnerhöhung, obwohl durch diesen Prozess Kosteneinsparungen für den Betrieb möglich werden (Yasukawa 2018). Insofern ist das gesellschaftskritische Element der Anerkennung numeraler Praktiken noch ausbaufähig. Es lassen sich folgende Aussagen festhalten (vgl. Yasukawa et al. 2018):

- Mathematik ist in alltäglichen Praktiken unsichtbar.

- Numerale Praktiken sind variabel und kontingent.

- Numerale Praktiken sind immer auf lokale Gegebenheit und globale Diskursen bezogen (z. B. UNESCO).

- In numeralen Praktiken verknüpfen sich Mensch und Objekt.

- Numerale Praktiken enthalten ein politisches Element, sind insofern nicht neutral.

Im folgenden Schritt wollen wir auf Forschungsarbeiten aus dem deutschen Sprachraum schauen. Diese beziehen sich sowohl auf eher emanzipatorische Herangehensweisen als auch auf Large-Scale-Assessments. Letztere werden im Wesentlichen dazu verwendet, ungleiche Chancen und die Vulnerabilität spezifischer Bevölkerungsgruppen aufzuzeigen.

\section{Finanzielle Grundbildung: Der Kern finanzieller Allgemeinbildung}

Numeralität hat Überschneidungsbereiche mit Finanzieller Grundbildung. Exemplarisch ist hier das Projekt „Curriculare Vernetzung - Schuldnerberatung als Ausgangspunkt für Grundbildung“ (CurVe) zu nennen, aus dem ein Kompetenzmodell entstand (Mania und Tröster 2015). Das Modell setzt sich explizit von umfassenderer finanzieller Bildung ab. Finanzielle Bildung kann als Teil umfassenderer ökonomischer Bildung verstanden werden und bildet dabei einen Teil von Verbraucherbildung (Remmele 2016). Finanzielle Grundbildung betrifft jedoch ausdrücklich ,die existenziell basalen und unmittelbar lebenspraktischen Anforderungen alltäglichen Handelns und der Lebensführung in geldlichen Angelegenheiten“ (Mania und Tröster 2014). Diese sind in dem Modell in Form einer Matrix beschrieben, die sowohl Domänen finanzieller Grundbildung ausweisen (Haushalten, Geld und Zahlungsverkehr, Ausgaben und Kaufen, etc.) als auch spezifische Dimensionen (Wissen, Lesen, Schreiben, Rechnen). Dies folgt der Annahme, dass sich finanzielle Grundbildung nicht allein auf das Rechnen beschränken lässt, sondern auch Lese- und Schreibpraktiken sowie Wissensbestände umfasst.

Die Studie LEO 2018 - Leben mit geringer Literalität hat als Large-Scale-Assessment-Studie die Literalität im Sinne der Lese- und Schreibkompetenz von Deutsch sprechenden Erwachsenen erfasst (Grotlüschen et al. 2019b). Zwar enthält die Studie kein Numeralitäts-Assessment; der Themenbereich finanzieller Praktiken und Grundkompetenzen wurde jedoch durch eine Reihe von Fragen erfasst. Diese Fragen basieren zu einem überwiegenden Teil auf den theoretischen Vorarbeiten des CurVe-Projekts und bilden die dort beschriebenen Kompetenzdomänen ab. Auf Basis von Selbsteinstufungen sind so Aussagen über finanzbezogene Praktiken und Grundkompetenzen möglich. Hinsichtlich der finanzbezogenen Praktiken zeigen sich literalitätsbezogene Einschränkung vor allem dann, wenn sie die Nutzung des Internets 
implizieren, etwa bei der Nutzung von Online-Banking oder bei der Informationssuche im Vorfeld größerer Anschaffungen. Hinsichtlich der selbsteingeschätzten funktional-pragmatischen und vor allem kritisch-hinterfragenden Grundkompetenzen zeigen sich systematische Unterschiede zwischen gering literalisierten Erwachsenen und der Gesamtbevölkerung: Gering literalisierte Erwachsene schreiben sich selbst signifikant geringere Kompetenzen bei der Auswahl von geeigneten Dienstleistungen (Telefonanbieter, Stromanbieter, Altersvorsorge) oder in Steuerfragen zu. Das gilt auch für den kritischen und reflektierten Umgang mit Online-Banking oder Ratenkäufen.

\section{Ergebnisse aus dem Hamburger Numeracy Projekt}

Das Hamburger Numeracy Projekt (2017-2020) erstellt Sekundäranalysen auf Basis bevölkerungsrepräsentativer Kompetenzstudien (TIMSS, IALS und PIAAC), ergänzt diese um UNESCO-Studien (RAMAA, GAML) sowie Arbeiten der Weltbank (STEP). Dies dient dazu, numerale Kompetenzen (Klieme und Hartig 2008) und numerale Praktiken (Lave 1988) zu untersuchen. Weiterhin werden numerale Praktiken vulnerabler Gruppen in qualitativen Analysen genauer betrachtet (im Falle von Flucht und Asyl, bei Überschuldung, im Alter, bei eingeschränkter Gesundheit oder bei Behinderung). Die historische Dimension wird mit Zeitzeugeninterviews der Nachkriegsgeneration exploriert.

\subsection{Unzureichende und absinkende numerale Kompetenzen}

England und Frankreich, die in den jeweils bevölkerungsrepräsentativen IVQ- und Skills-for-Life-Studie neben Literalität auch Numeralität getestet haben, berichten von einer Verschlechterung im Bereich Alltagsmathematik bei gleichzeitiger Verbesserung der Lesekompetenzen (Jonas 2012; Department for Business, Innovation and Skills (BIS) 2012).

Die 2013 publizierte PIAAC-Studie (Rammstedt 2013) differenziert das Bild. Insgesamt erreichen deutsche Erwachsene im internationalen Vergleich in der $\mathrm{Nu}-$ meralität bessere Leistungen als im Lesen. Die guten Leistungen der Erwachsenen sind jedoch durch den leistungsstärksten Teil der Bevölkerung hervorgerufen (Zabal et al. 2013, S. 57). Der Anteil der Deutschen auf und unter Kompetenzstufe I liegt bei $18 \%$ der Bevölkerung (ebd., S. 54f.). Zudem gehen diese Kompetenzen bei fehlendem Gebrauch leicht verloren (Bynner und Parsons 1998; NRDC Institute of Education 2010, S. 35).

Eben dieser praktische Gebrauch literaler und numeraler Kompetenzen scheint zudem im Rahmen des International Adult Literacy Surveys (IALS) Mitte der 1990er Jahre abzusinken (Desjardins 2017; Redmer und Dannath, im Druck).

\subsection{Ungleiche Erträge numeraler Kompetenzen}

Insgesamt führen Grundkompetenzen wie Literalität und Numeralität zu Erträgen (,Outcomes“), etwa im Bereich von Einkommen und Arbeitsplatzsicherheit (OECD 
2013; Vogtenhuber 2014) sowie sogenannten wider benefits of learning, etwa Gesundheit und sozio-politische Teilhabe (Bynner et al. 2003; Schuller 2017). Numeralität korreliert zudem höher mit den Einkommen als Literalität (Schleicher 2013). Diesen übergreifenden und wiederkehrend belegten Befund ergänzen und differenzieren verschiedene von Einzelstudien (Redmer et al. 2018; Liu et al. 2019). Die Befundlage ist uneinheitlich: Die OECD weist übergreifend hohe Erträge von $\mathrm{Nu}-$ meralität hinsichtlich der Einkommen aus, allerdings greifen Einkommenserträge weniger für Frauen als für Männer (Heilmann im Druck). Innerhalb derer, die hohe Schulabschlüsse erreicht haben, zeigen sich keine Effekte von Numeralität beim Eintritt in das Studium. Über numerale Kompetenzen hinaus ist eine Korrelation von Motivation und Zielstrebigkeit mit Einkommen zu konstatieren. Die allgemein konstatierten Erträge von Numeralität sind durch die Einzelstudien insofern zu differenzieren - nicht alle profitieren gleichermaßen von hoher Numeralität, andererseits sind neben Numeralität auch Zielstrebigkeit und Motivation relevant.

\subsection{Bedeutung numeraler Praktiken}

Ein Meilenstein der Forschung liegt in der Ausweitung der PIAAC-Erhebung über die Kompetenzmessung hinaus. Seit der ersten PIAAC-Runde 2012 werden unter anderem verschiedene numerale Praktiken erfragt. Gemäß Technical Report (OECD 2016) erfolgt dies über die folgenden Fragen:

Wie oft haben Sie normalerweise ...

... Rechnungen, Bankauszüge oder Ähnliches gelesen?

... Diagramme, Pläne, Karten oder Schaubilder gelesen?

... Preise, Kosten oder Budgets berechnet?

... Brüche, Dezimal- oder Prozentangaben verwendet oder berechnet?

... einen Taschenrechner verwendet, egal ob als eigenständiges Gerät oder im Computer?

... Diagramme, Schaubilder oder Tabellen erstellt?

... einfache Formeln oder Ähnliches verwendet?

... höhere Mathematik oder Statistik verwendet, wie zum Beispiel Analysis, komplexe Algebra, Trigonometrie oder Regressionsanalysen?

Eine Reihe komplexer Analysen zur Rolle (literaler und) numeraler Praktiken sind inzwischen publiziert. So erstellten Nienkemper und andere mithilfe einer latenten Klassenanalyse drei Kompetenznutzungsprofile, die neben schriftsprachlichen Praktiken auch Computernutzung und - in Grenzen - numerale Praktiken enthalten. Deutlich wird, dass die latente Klasse der so genannten ubiquitär schreibenden Erwachsenen auch ubiquitär kalkuliert ${ }^{7}$ (Nienkemper und Grotlüschen 2019), und zwar sowohl beruflich als auch privat. Das heißt: Erwachsene dieser Gruppe befassen sich permanent und an allen Orten damit, schriftlich zu kommunizieren. Das trifft

\footnotetext{
7 Verwendet wurden die Variablen mit der jeweils höchsten Streuung innerhalb der gering literalisierten Erwachsenen. Tatsächlich wurde bei der Auswertung aber versäumt, den Eigenwert numeraler Praktiken zur Geltung zu bringen. Die zwei verwendeten Variablen sind die Nutzung von Taschenrechner oder Kalkulationsprogramm am Arbeitsplatz sowie die Berechnung von Preisen und Budgets im Alltag. Interessanterweise sind eben diese breit streuenden Variablen auch in anderen Analysen aussagekräftig (Grotlüschen et al. 2019a).
} 
möglicherweise auch für numerale Praktiken zu, beispielsweise bezüglich der Einschätzung zeitlicher und finanzieller Ressourcen, effizienter Vorgehensweisen und relevanter Größenordnungen.

Während viele Erhebungen die Bedeutung numeraler Praktiken hervorheben (Reder 2017), zeigt der Trendvergleich numeraler Praktiken mithilfe der deutschen IALS-Daten und ausgewählter, vergleichbarer PIAAC-Daten ein Absinken numeraler Praktiken im Verlauf der letzten dreißig Jahre (Redmer und Dannath im Druck). Die Analysen wurden nach Berufsgruppen unterschieden und belegen eine Polarisierung des Arbeitsmarkts in fast allen betrachteten Ländern. Das bedeutet, dass sich mittlere Berufsgruppen aufteilen zugunsten größerer Anteile in höherqualifizierten und auch in niedrigqualifizierten Berufen (Redmer und Dannath im Druck). Parallel dazu finden sich in allen drei Gruppen gesunkene Werte für berufsbezogene numerale Praktiken. ${ }^{8}$

Dieses Ergebnis lässt sich vor dem Hintergrund des Verschwindens der Mathematik in der Technologie bei gleichzeitiger Dominanz von Mathematik in allen Lebensbereichen und fortbestehender Entwertung von Mathematik im täglichen Leben interpretieren. Dass Mathematik zunehmend unsichtbar wird, kann auch von Niss (2002, S. 371) belegt werden. Das Verschwinden der Mathematik in der Technologie zeigt demgegenüber Gellert mit dem Begriff „Demathematisierung“ (Gellert und Jablonka 2007).

Zusammenfassend lässt sich feststellen, dass die quantitativen Sekundäranalysen repräsentativer Datensätze, die numerale Kompetenzen bearbeiten, sowohl die Vorzüge höherer Kompetenzen ausweisen können (Liu et al. 2019) als auch Ungleichheit und Abdrängungsprozesse aufzeigen (Heilmann im Druck). Die Befundlage zu numeralen Praktiken zeigt wiederum, dass diese sowohl in höheren als auch in mittleren und gering qualifizierten Berufsgruppen seit Mitte der 1990er Jahre nachgelassen haben (Redmer und Dannath im Druck). Zugleich zeigt sich, dass nicht von allen Gruppen gleichermaßen ubiquitär mit Numeralität umgegangen wird (Nienkemper und Grotlüschen 2019).

\subsection{Vulnerabilität und Numeralität}

Unter „Vulnerabilität“ kann einerseits die Verletzbarkeit gesellschaftlicher Gruppen verstanden werden, die zwar noch teilhaben, sich jedoch in erhöhter Gefahr des Ausschlusses befinden (Streich 2009). Im feministischen Diskurs wird Vulnerabilität andererseits als conditio humana (Butler 1998) angesehen; allerdings gibt es auch hier Gruppen, die in höherem Ausmaß prekarisiert sind und daher höhere Vulnerabilität aufweisen (Janssen 2017).

\footnotetext{
${ }^{8}$ Da es sich um zwei Datensätze handelt (IALS und PIAAC) und zudem die Formalbildungsdaten und demzufolge die Kompetenzdaten des deutschen IALS-Datensatzes schadhaft sind, ist es nur möglich, die Werte nebeneinander zu stellen. Signifikanztests würden eine Integration des Datensatzes erfordern. Davon haben Autorin und der Autor aus den genannten Gründen abgesehen.
} 


\subsubsection{Mathematische Bedarfe und Selbstvertrauen bei Geflüchteten}

Die Situation geflüchteter Jugendlicher und Erwachsener beschäftigt sowohl die Bildungsforschung als auch die -praxis. Wenig ist bisher über die numeralen Kompetenzen und Praktiken nach Deutschland Geflüchteter bekannt. Allerdings gibt es in der (Schul-)Mathematik internationale Leistungsvergleichsstudien (TIMSS) aus den Jahren 2007, 2011 und 2015, an denen u. a. Herkunftsländer wie Syrien und Iran teilgenommen haben (Lüssenhop und Kaiser im Druck, S. 8). Weiterhin sind Nachbarländer (Türkei, Libanon, Jordanien) sowie Aufnahmeländer (Italien, Deutschland, Schweden) beteiligt.

Die Studien fokussieren zunächst einmal mathematische Kompetenzen und zeigen, dass die geringere finanzielle Ausstattung der Bildungssysteme in den so genannten Middle-Eastern- and North-African-Staaten mit unterdurchschnittlichen schulmathematischen Kompetenzen einhergehen (ebd.). Das bedeutet auch, dass niemand aus der Gruppe der syrischen und jordanischen Schülerinnen und Schüler der Repräsentativbefragungen das höchste Level in TIMSS erreicht hat (ebd., S. 20). Grundsätzlich haben diese nunmehr erwachsenen Personen eine Kenntnis von Ganzzahlen und Dezimalzahlen, Operatoren und basalen Grafiken, jedoch kein darüber hinausreichendes mathematisches Wissen (ebd.). Es ist also notwendig, hier anschließende Bildungsangebote vorzuhalten. Dies kann auf sehr fruchtbaren Boden fallen, denn die Einstellungen und die Wertschätzung für das Erlernen von Mathematik, ebenso wie das berichtete Selbstvertrauen hinsichtlich Mathematik in Iran, Jordanien, Libanon und Syrien fallen sogar höher aus als in den Vergleichsländern (ebd.). Die 2011 Befragten wären heute Anfang zwanzig und fallen in die größte Altersgruppe der Fluchtmigration nach 2015 (ebd., S. 8). Besonders für die Einmündung in Berufsbildung und Arbeitsmarkt ist eine entsprechende Qualifizierung vonnöten.

\subsubsection{Gering qualifizierte Beschäftigung: Denumeralisierung im Beruf?}

Redmer und Dannath (im Druck) weisen Beschäftigte im niedrig qualifizierten Sektor als vulnerabel aus und zeigen, dass die Arbeitsmarktanteile dieser Gruppe keineswegs verschwinden. Zudem zeigen sie, dass diese Gruppe über drei Jahrzehnte hinweg systematisch seltener (a) Diagramme und Tabellen verwendet hat. Auch (b) Rechnungen, Bankauszüge oder Budgettabellen werden seltener verwendet als in anderen Berufsgruppen. Umgekehrt kalkulieren gering qualifiziert Beschäftigte im Vergleich zu mittleren Qualifikationen am Arbeitsplatz heute etwas häufiger

(c) Budgets oder Kosten. Beschäftigte in gering qualifizierten Tätigkeiten haben insofern in zwei von drei Segmenten (a-b) eher mit ,Denumeralisierung“ zu tun, im dritten Segment (c) trifft das den Zeitvergleich, nicht den Berufsgruppenvergleich (ebd.).

\subsubsection{Tight Budget Control? Je weniger Geld, desto mehr wird gerechnet}

Tatsächlich zeigt sich auch an anderen Stellen, dass der Umgang mit alltäglichen Budgets im vulnerablen Segment der Bevölkerung eher verbreiteter ist als in besser 
situierten Gruppen. Betrachtet man Arbeitslosigkeit, Wohnungslosigkeit oder Überschuldung, so zeigt die jeweils monetär vulnerablere Gruppe eine häufigere Aktivität bei Budgets und Preisvergleichen. Enge Budgets (,tight budgets“) werden häufiger kontrolliert (,tight control“) (Grotlüschen et al. 2019a) als von der Vergleichsbevölkerung. Diese Ergebnisse fußen auf PIAAC-Daten sowie Ergänzungserhebungen im Bereich der Schuldnerberatung und Wohnungslosenhilfe. Auch am Übergang in das Rentenalter lassen sich phasenweise höhere numerale Aktivitäten beobachten, wie Redmer auf Basis von PIAAC und CiLL-Daten zeigt (Redmer und Grotlüschen 2019). Dabei sind ältere Frauen stärker mit der alltäglichen Bewirtschaftung des Haushalts betraut, während ältere Männer sich vermehrt um Bankangelegenheiten kümmern (ebd.). Die Existenz von Kindern erhöhen die Intensität numeraler Praktiken, auch wenn sie nicht mehr im Haushalt leben (ebd.).

\subsubsection{Numeracy Avoidance? Die Sicht der Beratung von Überschuldeten}

Nimmt man nunmehr die Seite der Beratenden bei Überschuldung in den Fokus, entsteht ein Widerspruch. Qualitative Gruppeninterviews zeigen, dass Beratende eine „numeracy avoidance“ (Angermeier und Ansen im Druck) ihrer Ratsuchenden feststellen. Diese hätten nur selten einen Überblick über ihre Einnahmen und Ausgaben (ebd., S. 10). Laut Schuldnerberatung äußerten überschuldete Ratsuchende, „sie bräuchten gar nicht anzufangen, etwas zusammenzuzählen; es bringe ja nichts“ (ebd.).

Widersprüche in der Befundlage könnten sich eventuell dadurch aufklären, dass solche Vermeidungsaussagen als Schutzäußerungen zu werten sind, die zwar Abneigung zum Ausdruck bringen, nicht aber mit dem realen Vermeiden numeraler Praktiken einhergehen. Wer also sagt, er bräuchte seine monatlichen Rückzahlungsverpflichtungen gar nicht erst zusammenzuzählen, kann dennoch sehr genau sein Budget im Blick haben und täglich Preise vergleichen. Darüber hinaus kann es sein, dass das Beratungspersonal ein unzutreffendes Bild ihrer Adressatinnen und Adressaten artikuliert. Hierzu bedarf es weiterer Forschung.

Das Beratungspersonal in der Überschuldungsberatung weist weiterhin darauf hin, dass Anbieter eine Art der Produktbeschreibung vorlegen, die Preisvergleiche gezielt erschwert. Genannt werden hier Kreditangebote, Ratenkäufe und Inkassoangebote, weiterhin Telefon- und Stromversorgung. Diese Kritik äußern auch Evans, Yasukawa, Mallows und Creese (Evans et al. 2017). Komplexe finanzielle Entscheidungen (Altersvorsorge, Steuererklärungen) trauen sich gering literalisierte Erwachsene entsprechend seltener zu als der Durchschnitt (Grotlüschen et al. 2019b).

\subsubsection{Habitus und Numeralität bei Älteren: Sparsamkeit als Tugend}

Der statistisch sichtbare Umgang mit dem Renteneintritt (Redmer und Grotlüschen 2019) zeigt sich auch im qualitativen Interview mit 64- bis 93-Jährigen (Zeuner et al. im Druck). Der Renteneintritt stellt neue Herausforderungen. Dies führt zu privater Buchhaltung und systematischem Sparen (für neue Brillengläser oder Unvorhergesehenes). Die befragten Generationen und sozialen Gruppen äußern, dass Sparsamkeit und ein Auskommen ohne Schulden zu ihrem Wertesystem gehöre 
(ebd., S. 7): „In their view, the virtue of the old days was not to spend more money than one has" (ebd., S. 8). Diese „Tugend“ lässt sich mit Bourdieu als integrierten Teil des Habitus bewerten (Bourdieu 1987); sie ist gemäß Habitustheorie jedoch nicht nur generationen- sondern auch sozialstatusspezifisch.

Gesundheitsbelange erzeugen zudem Wahrscheinlichkeitsabwägungen (Heilungschancen und Risiken einer Operation), skalierte Selbstbeobachtungen (Schmerzprotokolle) und Dosierungsaufgaben. Darüber hinaus werden Kosten und Boni bei Krankenversicherungen geltend gemacht (Zeuner et al. im Druck). Zeitfragen spielen besonders dann eine Rolle, wenn Betreuungsdienste das Haus zu vereinbarten Zeiten betreten.

Die Kombination aus finanzieller und gesundheitlicher Vulnerabilität hat Folgen für die soziale Einbindung respektive die Gefühle von Einsamkeit. Numeralität kann somit wenigstens in gewissem Ausmaß einen positiven Einfluss auf finanzielle Auskömmlichkeit und gesundheitliches Wohlergehen reklamieren, sie erhöht damit auch die Chance auf soziale Eingebundenheit in höherem Lebensalter. Genau dieser kausale Zusammenhang gibt den nachfolgend skizzierten Ergebnissen von Analysen aus querschnittlich angelegten Repräsentativerhebungen hohe Relevanz. Umgekehrt bestätigen die Repräsentativdaten die qualitativen Ergebnisse.

\subsubsection{Numerale Praktiken und subjektives Gesundheitsempfinden}

Höheres Alter führt zu umfassenderen gesundheitlichen Herausforderungen. Gesundheitsbezogene Handlungen und Entscheidungen basieren zum Teil auf numeralen Kompetenzen, unter anderem hinsichtlich medizinischer Dosierungen, aber auch bezüglich gesundheitsrelevanter Informationen auf Lebensmittelverpackungen oder in Ernährungstabellen. Das Verständnis von Chancen und Risiken einer Behandlung erfordert die Fähigkeit, Prozent- oder Wahrscheinlichkeitsangaben nachzuvollziehen.

Der Forschungsstand besagt inzwischen, dass literale Fähigkeiten die Zusammenhänge mit subjektivem Gesundheitsempfinden und Verhalten nicht hinreichend aufklären (Heilmann im Druck). Insofern wird inzwischen von „Gesundheitsnumeralität“" gesprochen. Eine solche Health Numeracy stellt den Grad dar, in dem Personen die Fähigkeit aufweisen, sich numerale, quantitative, grafische, biostatistische und wahrscheinlichkeitstheoretische Gesundheitsinformationen zugänglich zu machen, sie zu verarbeiten, zu interpretieren, zu kommunizieren und daran orientiert zu handeln (Golbeck et al. 2005, S. 375).

Basierend auf der Erweiterungsstudie PIAAC-L wurde regressionsanalytisch der Zusammenhang von numeralen Kompetenzen, Praktiken und subjektivem Gesundheitszustand sekundäranalytisch ausgewertet (Heilmann im Druck). Dabei wurde der Zusammenhang zwischen Gesundheitsverhalten (Nichtrauchen, Bewegung, gesundheitsbewusste Ernährung) und numeralen Praktiken auch unter Kontrolle soziodemografischer Variablen bestätigt. So genannte „komplexe numerale Praktiken“ (ebd., S. 13) wie die Interpretation statistischer Daten korrelieren mit allen drei Varianten gesunden Verhaltens. Eher „grundlegende numerale Praktiken“, wie das Abschätzen von Messungen und die Berechnung einfacher Durchschnittswerte, korrelieren jeweils mit einigen der drei Variablen gesunden Verhaltens (ebd.). 
Die Daten zeigen, dass sich die Ausweitung von Daten und Statistiken in alle Lebensbereiche, so auch im Gesundheitsbereich, neue Herausforderungen an die Menschen mit sich bringt.

\subsubsection{Sichtbarkeit herstellen: Numerale Praktiken bei behinderten Menschen}

Zeuner, Pabst und Benz-Gydat (Zeuner et al. im Druck) zitieren einen 92-jährigen Interviewpartner mit den Worten:

Today I am handicapped and [...] if I could still get out now [...] things would be different. Then I could take part in life outside and go there, go to a concert.

But all that doesn't work anymore.

Der Interviewte ist auf ambulante Pflege angewiesen. Seine Alterseinschränkungen werden üblicherweise nicht als Behinderung angesehen. Einen menschenrechtsbasierten Ansatz vertritt dagegen Hirschberg (2014; Hirschberg et al. 2019). Demnach würden auch altersbedingte Behinderungen unter die Behindertenrechtskonvention fallen. Ein Teilhabeausschluss, wie oben berichtet, wäre dann sogar rechtswidrig (Art. 3, Abs. 3, GG).

Davon unabhängig konnten Schreiber-Barsch, Curdt und Gundlach (SchreiberBarsch et al. im Druck) feststellen, dass elf teilnehmend begleitete Personen mit Lernschwierigkeiten in allen Dimensionen numeraler Praktiken aufweisen. Die Autorinnen spiegeln die erlebten numeralen Praktiken gegen etablierte Kompetenzrahmen aus dem Bereich der Erwachsenen-Numeralität sowie aus dem Bereich der Schulmathematik. Das umfasst Kompetenzrahmen des Deutschen Volkshochschulverbands, der KMK sowie das Numeracy-Theoriekonzept der PIAAC-Studie. Sie zeigen, dass die Studienteilnehmenden Fragen von Raum und Form, Zahlen und Kalkulation, Größen und Maßen, Hilfsmitteln (Listen, Computer, Navigationssysteme), Muster und Strukturen, Wahrscheinlichkeiten, Häufigkeiten und Chancen bearbeiten und bewältigen. Personen mit Lernschwierigkeiten sind in Large-Scale-Assessments unterrepräsentiert. Schreiber-Barsch et al. argumentieren, dass Praktiken behinderter Menschen erst Sichtbarkeit und gesellschaftliche Anerkennung finden, wenn sie auch in Messungen auftauchen. Auch Hirschberg fordert eine entsprechend verbesserte Datenlage (Hirschberg 2014).

\subsubsection{Adult Numeracy auf dem gesamten Globus? Sustainable Development Goal}

Die Sustainable Development Goals wurden 2015 von den Vereinten Nationen verabschiedet. Das vierte der siebzehn Nachhaltigkeitsziele fordert:

Für alle Menschen inklusive, chancengerechte und hochwertige Bildung sowie Möglichkeiten zum lebenslangen Lernen sicherstellen.

Dieses Ziel untergliedert sich in sieben Teilziele, die auf Schulbildung, frühkindliche Bildung, Berufs- und Hochschulbildung, Kompetenzen für gute Arbeit, Gendergerechtigkeit und Inklusion, universale Literalität und Numeralität sowie Global Citizenship abzielen. Numeralität kann dieselbe Relevanz beanspruchen wie Literalität: 
By 2030, ensure that all youth and a substantial proportion of adults, both men and women, achieve literacy and numeracy.

Hier greift nun die Monitoring-Initiative des UNESCO-Instituts für Statistik sowie des UNESCO-Instituts für Lebenslanges Lernen (Ziel 4.6.1) unter dem Titel „Global Alliance for Monitoring Learning“‘. Dieser Prozess nutzt verfügbare Bildungsstatistiken für die Berichte zur Indikatorenerreichung. Dabei entstehen aus OECD-Studien zunehmend Derivate, die sich auf Länder des globalen Südens richten, z. B. PISA und PISA for Development sowie PIAAC und STEP (World Bank 2014). Allerdings leitet sich daraus nun die Frage ab, welche Erhebungen in Ländern geringer oder mittlerer Einkommen sinnvoll genutzt oder erfasst werden können. Hier tut sich ein Dilemma auf: Bildungsdaten sind notwendig, um Ressourcen zu aggregieren, auch für das notorisch unterfinanzierte lebenslange Lernen (Singh 2019). Zugleich entsteht ein Testmonopol, das sich auf weltweit durchgesetzte Definitionen, Skalen und Instrumente stützt (Addey 2018; Grotlüschen et al. 2019c). Zwei Gruppen von Expertinnen und Experten haben entsprechende Empfehlungen vorgelegt. $\mathrm{Zu}$ berichten ist nach einer 2019 abgehaltenen Beratung zur UNESCO-Strategie zu Youth and Adult Literacy 2020-2025 der Ansatz, eher die Diversität von Assessments als einen erweiterten Monopolismus von Assessments zu unterstützen.

\section{Hohe Relevanz, sinkender Gebrauch, hohe Vulnerabilität}

Deutlich wird bei der Zusammenschau der Befunde, dass Numeralität eigenständige Effekte auf Erwerbstätigkeit, Einkommen, Führungspositionen und Weiterbildungsbeteiligung hat. Numeralität ist insofern von hoher Bedeutung, wird aber gegenüber Literalität deutlich seltener beforscht (Carpentieri et al. 2009). Auch die gleichlautende Relevanz in den Nachhaltigkeitszielen spiegelt sich nicht in gleichermaßen intensiven Beratungen zu Numeralität wider. Dennoch müsste sich der Aufbau numeraler Kompetenzen für Individuum und Gesellschaft auszahlen, wie die quantitativen Analysen zeigen. Allerdings zeigt sich auch hier eine ungleich verteilte Vulnerabilität gesellschaftlicher Gruppen. Sowohl Überschuldete als auch Wohnungslose, Arbeitssuchende, Geflüchtete und gering qualifiziert Beschäftigte können zudem als vulnerable Gruppen gelten. Hinzu treten Geschlecht, Alter, Migrationshintergrund, Gesundheit, Behinderung und der sozioökonomische Status der Herkunftsfamilie.

Qualitative Daten weisen darauf hin, wie wichtig numerale Praktiken für die Durchsetzung eigener Rechte und Verfügungsmöglichkeiten in Beruf und Gesellschaft sind. Dieses Ergebnis ist in unterschiedlichsten gesellschaftlichen Kontexten und Ländern replizierbar. Gleichzeitig bestätigt sich der Befund einer sukzessive hinter der Technologie verschwindenden oder bereits unsichtbaren Numeralität und zwar sowohl hinsichtlich der Kompetenzen als auch der Praktiken.

Wenn nunmehr aber die Relevanz numeraler Kompetenzen und Praktiken facettenreich belegt ist, dann erscheint der Rückgang numeraler Kompetenzen (Jonas 2012) und Praktiken (Redmer und Dannath im Druck) problematisch. Daraus entsteht ein Auftrag an Bildungspolitik und Praxis, zur verbesserten Numeralität 
Erwachsener beizutragen. Gerade in Zeiten zunehmender Quantifizierung und Datafizierung kann es hier Bildungsbedarfe geben.

\section{Desiderata}

Wissenschaftlicher Handlungsbedarf zeigt sich zunächst hinsichtlich der verbesserten theoretischen Erkundung und Modellierung von Numeralität im Rahmen der Grundbildung Erwachsener. Wenn Numeralität nicht nur aufgrund humanistischnormativer Annahmen, sondern auch aufgrund der Befundlage eine ähnliche Relevanz haben soll wie Literalität, dann ist sie begrifflich auch in den Konstrukten von Grundbildung zu verankern und zu differenzieren. $\mathrm{Zu}$ fragen ist dann, ob es beispielsweise neben Financial Literacy, Digital Literacy, Health Literacy, Political Literacy auch einschlägige Ansätze zur Numeracy geben soll.

Erste Ansätze dafür liegen vor, etwa wird im CurVe-Projekt vom „Rechnen im Bereich Finanzieller Grundbildung“ gesprochen. ${ }^{9}$ Ein Übergang zum Begriff „Financial Numeracy“ läge nahe. Parallel zur Health Literacy gibt es, wie oben referiert, bereits einen Begriff Health Numeracy. Digital Numeracy ist in Zeiten von Datafizierung und Algorithmisierung vermutlich zutreffender als Digital Literacy. Und Political Numeracy müsste als Ausgangspunkt die Ansätze der Critical Numeracy (Geiger et al. 2015a) oder Statistical Literacy (Ridgway et al. 2019) nehmen.

Weiterhin wird in der Literalitätsforschung seit Langem über Stereotypisierungen und Stigmatisierungen, Defizitorientierung und das „blaming the victim“ diskutiert (Belzer und Pickard 2015; Bittlingmayer 2008). Die Adressatenkonstruktion gering numeralisierter Erwachsener wird demgegenüber nur in der Schuldnerberatungsforschung erkennbar thematisiert.

Darüber hinaus ist der deutsche, aber auch der internationale quantitative Datenbestand zur Numeralität Erwachsener noch nicht umfassend ausgeschöpft. Längsschnittdaten, international vergleichende Daten, Daten zu digitalen und numeralen Kompetenzen liegen seit 2013 vor. LEO-Daten zur finanziellen und digitalen Grundbildung - die immerhin Überschneidungsbereiche mit Numeralität aufweisen - sind seit 2018 vorhanden. Zunehmend werden auch qualitative Daten für Sekundäranalysen bereitgestellt. Hier können der Literalitätsforschung zugeordnete Interviewund Beobachtungstranskripte hinsichtlich nicht identifizierter numeraler Praktiken re-analysiert werden.

Die bisherige Befundlage zeigt: Numeralität wird von Erwachsenen nicht als solche wahrgenommen, sie verschwindet in der Technologie und sie ist dennoch wichtig für politische und berufliche Prozesse. Algorithmisierung, Datafizierung, Digitalisierung und Quantifizierung in einer so genannten Daten- oder Kontrollgesellschaft werden mit Bezug auf Numeralität und Literalität diskutiert (Evans et al. 2019; Smythe 2018) und rahmen sie in neuer Weise. Hier ergeben sich noch vielfältige Forschungsperspektiven, die derzeit noch nicht absehbar sind.

\footnotetext{
9 www.die-curve.de.
} 
Open Access Dieser Artikel wird unter der Creative Commons Namensnennung 4.0 International Lizenz (http://creativecommons.org/licenses/by/4.0/deed.de) veröffentlicht, welche die Nutzung, Vervielfältigung, Bearbeitung, Verbreitung und Wiedergabe in jeglichem Medium und Format erlaubt, sofern Sie den/die ursprünglichen Autor(en) und die Quelle ordnungsgemäß nennen, einen Link zur Creative Commons Lizenz beifügen und angeben, ob Änderungen vorgenommen wurden.

\section{Literatur}

Abraham, E., \& Linde, A. (2010). Alphabetisierung/Grundbildung als Aufgabengebiet der Erwachsenenbildung. In R. Tippelt \& A. von Hippel (Hrsg.), Handbuch Erwachsenenbildung/Weiterbildung (4. Aufl. S. 889-903). Wiesbaden: Springer.

Addey, C. (2018). Assembling literacy as global: the danger of a single story. In M. Milana, J. Holford, S. Webb, P. Jarvis \& R. Waller (Hrsg.), The Palgrave international handbook of adult and lifelong education and learning (S. 315-335). London: Palgrave Macmillan.

Agence Nationale de la Lutte contre l'Illettrisme (2005by). Illiteracy: the statistics: analysis by the national agency to fight illiteracy of the IVQ survey conducted in 2004-2005 by INSEE. Lyon: ANLCI.

Angermeier, K., \& Ansen, H. (im Druck). Value and understanding of numeracy practices in German debt counselling from the perspective of professionals. ZDM Mathematics Education, 52(2).

Askew, M. (2015). Numeracy for the 21st century. A commentary. ZDM Mathematics Education, 47(4), 707-712. https://doi.org/10.1007/s11858-015-0709-0.

Barton, D., \& Hamilton, M. (2003). Literacy practices. In D. Barton (Hrsg.), Situated literacies. Reading and writing in context (S. 1-6). London: Routledge.

Belzer, A., \& Pickard, A. (2015). From heroic victims to competent comrades. Views of adult literacy learners in the research literature. Adult Education Quarterly, 65(3), 250-266. https://doi.org/10.1177/ 0741713615580015.

Bittlingmayer, U.H. (2008). Blaming, producing und activating the victim. Materialistisch inspirierte Anmerkung zu verdrängten Dimensionen sozial bedingter gesundheitlicher Ungleichheit. In G. Hensen \& P. Hensen (Hrsg.), Gesundheitswesen und Sozialstaat. Gesundheitsförderung zwischen Anspruch und Wirklichkeit 1. Aufl. Gesundheit und Gesellschaft. (S. 239-258). Wiesbaden: Springer.

Bourdieu, P. (1987). Die feinen Unterschiede. Kritik der gesellschaftlichen Urteilskraft. Frankfurt am Main: Suhrkamp.

Bundesministerium für Bildung und Forschung (BMBF) \& Kultusministerkonferenz (2016). Grundsatzpapier zur Nationalen Dekade für Alphabetisierung und Grundbildung 2016-2026. Den funktionalen Analphabetismus in Deutschland verringern und das Grundbildungsniveau erhöhen. www. alphadekade.de/files/01_GrundsatzpapierzurNationalenDekadeAlphabetisierungundGrundbildung_ final.pdf. Zugegriffen: 24. Sept. 2019.

Butler, J. (1998). Haß spricht. Zur Politik des Performativen. Berlin: Berlin-Verlag.

Bynner, J., \& Parsons, S. (1998). Use it or lose it? The impact of time out of work on literacy and numeracy skills. London: Basic Skills Agency.

Bynner, J., Schuller, T., \& Feinstein, L. (2003). Wider benefits of education: skills, higher education and civic engagement. Zeitschrift für Pädagogik, 49(3), 341-361.

Carpentieri, J.D., Litster, J., \& Frumkin, L. (2009). Adult numeracy: a review of research. www. nationalnumeracy.org.uk/sites/default/files/carpentieri_et_al_2009_bbc_adult_numeracy_a_review_ of_research.pdf. Zugegriffen: 24. Sept. 2019. London: National Research and Development Centre for Adult Literacy and Numeracy (NRDC).

Coben, D. (2003). Adult numeracy: review of research and related literature. https://dera.ioe.ac.uk/22487/ 1/doc_2802.pdf. Zugegriffen: 24. Sept. 2019. London: NRDC Institute of Education.

Conrad, A., Herzig, A., \& Kopitzsch, F. (Hrsg.). (1998). Das Volk im Visier der Aufklärung. Studien zur Popularisierung der Aufklärung im späten 18. Jahrhundert. Tagung „Popularisierung der Aufklärung im späten 18. Jahrhundert“. Hamburg: LIT.

Department for Business, Innovation and Skills (BIS) (2012). 2011 skills for life survey: a survey of literacy, numeracy and ICT levels in England. London (BIS research paper, 81). https:// assets.publishing.service.gov.uk/government/uploads/system/uploads/attachment_data/file/36000/ 12-p168-2011-skills-for-life-survey.pdf. Zugegriffen: 24. Sept. 2019.

Deschauer, S. (2013). Das Zweite Rechenbuch Von Adam Ries: Eine moderne Textfassung mit Kommentar und metrologischem Anhang und einer Einführung in Leben und Werk des Rechenmeisters. Wiesbaden: Vieweg und Teubner. 
Desjardins, R. (2017). Is literacy and the practice of literacy on the decline? In A. T. de Matos, G. d'Oliveira Martins \& P. Hanenberg (Hrsg.), The future within reach (S. 761-766). Lissabon: Catholic University of Portugal.

DfES (2003). The skills for life survey: a national needs and impact survey of literacy, numeracy and ICT skills. https://assets.publishing.service.gov.uk/government/uploads/system/uploads/attachment_ data/file/36000/12-p168-2011-skills-for-life-survey.pdf. Zugegriffen: 24. Sept. 2019. London: DfES Research Brief RB490.

Duncker-Euringer, C. (2017). Was ist Grundbildung? In B. Menke \& W. Riekmann (Hrsg.), Politische Grundbildung. Inhalte - Zielgruppen - Herausforderungen (S. 13-33). Schwalbach/Ts.: Wochenschau Verlag.

Effe, G., Fuchs, P., \& Kaiser, G. (1976). Lohnerhöhungen in mathematischer Behandlung. Unterricht im Konflikt, Konflikt im Unterricht. Bamberg: Schwarz.

Euringer, C. (2016a). Grundbildung im Spannungsfeld bildungspolitischer Ein- und Abgrenzungsinteressen. Zeitschrift für Weiterbildungsforschung, 39(2), 241-254. https://doi.org/10.1007/s40955-0160066-9.

Euringer, C. (2016b). Was ist „Grundbildung Erwachsener“? Das Grundbildungsverständnis der öffentlichen Bildungsverwaltung im Kontext von Interessen und Machtverhältnissen. Dissertation. Bielefeld: W. Bertelsmann.

Evans, J., Ruane, S., \& Southall, H. (Hrsg.). (2019). Data in society: challenging statistics in an age of globalisation. Bristol, UK: Policy Press.

Evans, J., Yasukawa, K., Mallows, D., \& Creese, B. (2017). Numerical skills and the numerate environment: affordances and demands. Adults Learning Mathematics. An International Journal, 12(1), 17-26.

Freire, P. (1996). Pädagogik der Unterdrückten. Bildung als Praxis der Freiheit. Reinbek bei Hamburg: Rowohlt.

Gal, I., Alatorre, S., Close, S., Evans, J., Johansen, L., Maguire, T., Manly, M., \& Tout, D. (Hrsg.). (2009). PIAAC numeracy. A conceptual framework. OECD Education Working Paper, Bd. 35. Paris: OECD Publishing.

Geiger, V., Forgasz, H., \& Goos, M. (2015a). A critical orientation to numeracy across the curriculum. ZDM: The International Journal on Mathematics Education. https://doi.org/10.1007/s11858-0140648-1.

Geiger, V., Goos, M., \& Forgasz, H. (2015b). A rich interpretation of numeracy for the 21st century. A survey of the state of the field. ZDM Mathematics Education, 47(4), 531-548. https://doi.org/10. 1007/s11858-015-0708-1.

Gellert, U., \& Jablonka, E. (2007). Mathematisation - demathematisation. In U. Gellert \& E. Jablonka (Hrsg.), Mathematisation and demathematisation. Social, philosophical and educational ramifications (S. 1-18). Amsterdam: Sense.

Golbeck, A.L., Ahlers-Schmidt, C.R., Paschal, A. M., \& Dismuke, S.E. (2005). A definition and operational framework for health numeracy. American Journal of Preventive Medicine, 29(4), 375-376. https://doi.org/10.1016/j.amepre.2005.06.012.

Grotlüschen, A. (2018). Grundbildung von Erwachsenen. In R. Tippelt \& B. Schmidt-Hertha (Hrsg.), Handbuch Bildungsforschung (S. 1261-1278). Heidelberg: Springer.

Grotlüschen, A., Buddeberg, K., Ansen, H., \& Redmer, A. (2019a). Vulnerable subgroups and numeracy practices. How poverty, debt and unemployment relate to everyday numeracy practices. Adult Education Quarterly. https://doi.org/10.1177/0741713619841132.

Grotlüschen, A., Buddeberg, K., Dutz, G., Heilmann, L. M., \& Stammer, C. (2019b). LEO 2018. Living with low literacy. Hamburg. http://blogs.epb.uni-hamburg.de/leo/files/2019/05/LEO-2018-firstresults.pdf. Zugegriffen: 24. Sept. 2019.

Grotlüschen, A., Mallows, D., Reder, S., \& Sabatini, J. (2016). Adults with low proficiency in literacy or numeracy. OECD Education Working Papers. https://doi.org/10.1787/5jm0v44bnmnx-en.

Grotlüschen, A., Thériault, V., Nienkemper, B., \& Capstick, T. (2019c). Critical viewpoints on adult literacy practices at the time of PIAAC. International Journal of Lifelong Education, 38(4), 361-365. https://doi.org/10.1080/02601370.2019.1614105.

Harding, C. (2011). 2011 skills for life survey: headline findings (BIS research paper, 57). https:// assets.publishing.service.gov.uk/government/uploads/system/uploads/attachment_data/file/623399/ 11-1367-2011-skills-for-life-survey-findings.pdf. Zugegriffen: 24. Sept. 2019.

Heilmann, L. (im Druck) Health and numeracy. The role of numeracy skills in health satisfaction and health-related behaviour. ZDM Mathematics Education, 52(2). 
Hinrichs, E. (1998). Zur Erforschung der Alphabetisierung in Nordwestdeutschland in der Frühen Neuzeit. In A. Conrad, A. Herzig \& F. Kopitzsch (Hrsg.), Das Volk im Visier der Aufklärung. Studien zur Popularisierung der Aufklärung im späten 18. Jahrhundert (S. 35-56). Hamburg: LIT.

Hirschberg, M. (2014). Ethische Richtlinien für Forschung und Wissenschaft. In E. Mührel (Hrsg.), Perspektiven sozialpädagogischer Forschung. Methodologien; Arbeitsfeldbezüge; Forschungspraxen (S. 347-380). Wiesbaden: Springer.

Hirschberg, M., Bonna, F., \& Stobrawe, H. (2019). Gelingensbedingungen zur Umsetzung eines Menschenrechts: Professionalisierung in der Inklusiven Erwachsenenbildung. weiter bilden, 1, 21-23.

von Humboldt, W. (1809). Ueber die mit dem Koenigsbergischen Schulwesen vorzunehmende Reformen Auszug aus: Der Königsberger und der Litauische Schulplan. In A. Flitner \& K. Giel (Hrsg.), Werke in fünf Bänden, Band IV, (S. 168-173). Darmstadt: Wissenschaftliche Buchgesellschaft.

Janssen, A. (2017). Verletzbare Subjekte. Dissertation. Opladen: Budrich Uni Press.

Jonas, N. (2012). Pour des générations les plus récentes, les difficultés des adultes diminuent à l'écrit, mais augmentend en calcul. www.insee.fr/fr/themes/document.asp?ref_id=ip1426. Zugegriffen: 24. Sept. 2019. Hrsg. v. INSEE. Paris (INSEE PREMIERE, 1426 Décembre 2012.

Jonas, N. (2018). Numeracy practices and numeracy skills among adults. Education working papers, Bd. 177. Paris: OECD.

Kaiser-Meßmer, G. (1986). Anwendungen im Mathematikunterricht. Dissertation. Bad Salzdetfurth: Franzbecker.

Kalman, J., \& Solares, D. (2018). Tear it out and rip it up or you might get charged again: Paying debts at the company store in a farm workers' camp in Mexico. In K. Yasukawa, A. Rogers, K. Jackson, \& B. V. Street (Hrsg.), Numeracy As Social Practice: Global and Local Perspectives (S. 59-75). Milton: Routledge.

Kane, P. (2018). Estimation by kiwifruit orchard managers and urban refuse/recycling operators within their situated horticultural or civic workplace practices: case studies from New Zealand. In K. Yasukawa, A. Rogers, K. Jackson \& B. V. Street (Hrsg.), Numeracy as social practice. Global and local perspectives (S. 21-39). Milton: Routledge.

Khuzwayo, H. (2018). "Occupation of our minds": a metaphor to explain mathematics education in South Africa in the apartheid era. In K. Yasukawa, A. Rogers, K. Jackson \& B. V. Street (Hrsg.), Numeracy as social practice. Global and local perspectives (S. 171-186). Milton: Routledge.

Klieme, E., \& Hartig, J. (2008). Kompetenzkonzepte in den Sozialwissenschaften und im erziehungswissenschaftlichen Diskurs. In M. Prenzel, I. Gogolin \& H. Krüger (Hrsg.), Kompetenzdiagnostik (S. 11-32). Wiesbaden: VS.

Kultusministerkonferenz Arbeitskreis Weiterbildung (2015). Nationale Strategie Alphabetisierung - Bericht 2015

Lave, J. (1988). Cognition in practice. Mind, mathematics, and culture in everyday life. Cambridge, New York: Cambridge University Press.

Lave, J. (1993). Textaufgaben im Mathematikunterricht. Mikrokosmos der Widersprüche zwischen schulischem Lernen und außerschulischer Lebenspraxis. FKP (Forum Kritische Psychologie), 31, 5-28.

Lave, J., \& Wenger, E. (1991). Situated learning. Legitimate peripheral participation. Cambridge, New York: Cambridge University Press.

Linde, A. (2008). Literalität und Lernen. Eine Studie über das Lesen- und Schreibenlernen im Erwachsenenalter. Münster: Waxmann.

Liu, H., Fernandez, F., \& Grotlüschen, A. (2019). Beyond literacy and numeracy skills - examining selfdirectedness in learning in Yunnan, Vietnam, Germany, and the United States. International Journal of Educational Development, 70(Article 102088). https://doi.org/10.1016/j.ijedudev.2019.102088

Lüssenhop, M., \& Kaiser, G. (im Druck) Numeracy in the context of forced migration - a secondary analysis of TIMSS data. ZDM Mathematics Education, 52(2).

Mania, E., \& Tröster, M. (2014). Finanzielle Grundbildung: Ein Kompetenzmodell entsteht. Hessische Blätter für Volksbildung, 2, 136-145.

Mania, E., \& Tröster, M. (2015). Finanzielle Grundbildung. Konzepte, Förderdiagnostik und Angebote. In A. Grotlüschen \& D. Zimper (Hrsg.), Literalitäts- und Grundlagenforschung (S. 45-60). Münster: Waxmann.

Menke, B., \& Riekmann, W. (Hrsg.). (2017). Politische Grundbildung. Inhalte - Zielgruppen - Herausforderungen. Schwalbach/Ts.: Wochenschau Verlag.

Nienkemper, B., \& Grotlüschen, A. (2019). Using PIAAC data to learn more about the literacy practices of adults. International Journal of Lifelong Education, 38(4), 393-405. https://doi.org/10.1080/ 02601370.2019 .1596171 . 
Niss, M. (2002). Mathematics in society. In R. Biehler, R. W. Scholz, R. Strässer \& B. Winkelmann (Hrsg.), Didactics of mathematics as a scientific discipline (S. 367-378). Dordrecht: Kluwer.

NRDC Institute of Education (2010). BBC raw numeracy literature review May 2010. http://downloads. bbc.co.uk/learning/learningoverview/impact_of_low_numeracy.pdf. Zugegriffen: 24. Sept. 2019.

Nthogo Lekoko, R., Mompoloki Suping, S., \& Pitso, O. (2018). Critical humanistic pedagogy in the context of adult basic education: making sense of numercy as social empowerment. In K. Yasukawa, A. Rogers, K. Jackson \& B. V. Street (Hrsg.), Numeracy as social practice. Global and local perspectives (S. 204-224). Milton: Routledge.

OECD (Hrsg.). (2016). Technical report of the survey of adult skills (PIAAC). Paris: OECD Publishing.

OECD (2013). OECD skills outlook 2013: first results from the survey of adult skills. Paris: OECD Publishing. https://doi.org/10.1787/9789264258051-en.

OECD and PIAAC Numeracy Expert Group (2009). PIAAC numeracy: a conceptual framework. OECD education working paper, Bd. 35.

Rammstedt, B. (Hrsg.). (2013). Grundlegende Kompetenzen Erwachsener im internationalen Vergleich. Ergebnisse von PIAAC 2012. Münster: Waxmann.

Reder, S. (2011). Some thoughts on IALS measurement validity, program impact, and logic models for policy development. Fall Institute 2011. Montreal: The Centre for Literacy.

Reder, S. (2017). Adults' engagement in reading, writing and numeracy practices. Applied linguistics faculty publications and presentations, Bd. 22. Portland: Portland State University.

Redmer, A., \& Grotlüschen, A. (2019). Alltagsmathematische Praktiken im höheren Lebensalter. Zeitschrift für Weiterbildungsforschung. https://doi.org/10.1007/s40955-019-0135-y.

Redmer, A., \& Dannath, J. (im Druck) Changes in employment since the 1990 s - trend of numeracy practices at work. ZDM Mathematics Education, 52(2).

Redmer, A., Heilmann, L., \& Grotlüschen, A. (2018). Grundkompetenzen und Hochschulzugang. Zur Rolle von Literalität und Numeralität beim Zugang zur Hochschule. Magazin erwachsenenbildung.at. Das Fachmedium für Forschung, Praxis und Diskurs (34). https://erwachsenenbildung.at/magazin/ 18-34/meb18_34.pdf. Zugegriffen: 24. Sept. 2019.

Remmele, B. (2016). Ökonomische Kompetenzen. Was sie umfassen und wie man sie misst. DIE Zeitschrift für Erwachsenenbildung, 1, 22-25.

Ridgway, J., Nicholson, J., Sutherland, S., \& Hedger, S. (2019). Critical statistical literacy and interactive data visualisations. In J. Evans, S. Ruane \& H. Southall (Hrsg.), Data in society. Challenging statistics in an age of globalisation (S. 349-358). Bristol: Policy Press.

Rogers, A., \& Street, B. V. (2018). Part I: Using case studies to expose the significance of what "surrounds" mathematics in numeracy practices. In K. Yasukawa, A. Rogers, K. Jackson \& B. V. Street (Hrsg.), Numeracy as social practice: global and local perspectives (S. 19-20). Milton: Routledge.

Schaeffer, D., Vogt, D., Berens, E., \& Hurrelmann, K. (2016). Gesundheitskompetenz der Bevölkerung in Deutschland. Ergebnisbericht. Bielefeld: Universität Bielefeld. www.uni-bielefeld.de/gesundhw/ag6/ downloads/Ergebnisbericht_HLS-GER.pdf. Zugegriffen: 24. Sept. 2019.

Schleicher, A. (2013). Skilled for life? Key findings from the survey of adult skills. https://www. slideshare.net/OECDEDU/skilled-for-life-key-findings-from-the-survey-of-adult-skills-andreasschleicher-special-advisor-to-the-secretarygeneral-on-education-policy-27212444. Zugegriffen: 24. Sept. 2019.

Schlögl, P. (2016). Kompetent! Aber wofür genau? Kulturkontakt Magazin, S. 6-9. (Winter 2016/2017)

Schreiber-Barsch, S., Curdt, W., \& Gundlach, H. (im Druck) Whose voices matter? Adults with learning difficulties and the emancipatory potential of numeracy practices. ZDM Mathematics Education, $52(2)$.

Schuller, T. (2017). What are the wider benefits of learning across the life course? Future of skills \& lifelong learning. http://dera.ioe.ac.uk/29773/1/Skills_and_lifelong_learning_-_the_benefits_of_adult_ learning_-_schuller_-_final.pdf. Zugegriffen: 24. Sept. 2019.

Singh, S. (2019). New Horizons for the Development Education in the Context of Sustainable Development Goals. International Journal of Research in Social Sciences, 9, 3(1), 326-341. http://www.ijmra.us/ project\%20doc/2019/IJRSS_MARCH2019/IJRSSMarch19.pdf

Smythe, S. (2018). Adult learning in the control society: digital era governance, literacies of control, and the work of adult educators. Adult Education Quarterly. https://doi.org/10.1177/0741713618766645.

Street, B. (2003). What's "new" in the New Literacy Studies. Critical approaches to literacy in theory and practice. Current Issues in Comparative Education, 5(2), 77-91.

Streich, W. (2009). Vulnerable Gruppen: „Verwundbarkeit“ als politik-sensibilisierende Metapher in der Beschreibung gesundheitlicher Ungleichheit. In K. Hurrelmann \& M. Richter (Hrsg.), Gesundheitliche Ungleichheit. Grundlagen, Probleme, Perspektiven (2. Aufl. S. 301-307). Wiesbaden: Springer. 
Tenorth, H. (2004). Stichwort: „Grundbildung“ und „Basiskompetenzen“. Herkunft, Bedeutung und Probleme im Kontext allgemeiner Bildung. Zeitschrift für Erziehungswissenschaft, 7(2), 169-182.

Tout, D. (1997). Some reflections on adult numeracy. In D. Coben (Hrsg.), Adults learning maths-3: proceedings of the third international conference of adults learning maths. London (S. 13-15).

Tröster, M. (Hrsg.). (2000). Spannungsfeld Grundbildung. Bielefeld: W. Bertelsmann.

Trumann, J. (2009). Bibliotheken als kommunale Lernzentren. In P. Faulstich \& M. Bayer (Hrsg.), Lernorte. Vielfalt von Weiterbildungs- und Lernmöglichkeiten (S. 47-64). Hamburg: VSA.

Tsatsaroni, A., \& Evans, J. (2014). Adult numeracy and the totally pedagogised society. PIAAC and other international surveys in the context of global educational policy on lifelong learning. Educ Stud Math, 87(2), 167-186. https://doi.org/10.1007/s10649-013-9470-x.

Vogtenhuber, S. (2014). Die berufliche Nutzung mathematischer Kompetenzen: Determinanten der Utilisierung und Effekte auf das Einkommen. In Statistik Austria (Hrsg.), Schlüsselkompetenzen von Erwachsenen - Vertiefende Analysen der PIAAC-Erhebung 2011/12 (S. 354-374). Wien: Statistik Austria.

Wolf, K. D., \& Koppel, I. (2017). Digitale Grundbildung: Ziel oder Methode einer chancengleichen Teilhabe in einer mediatisierten Gesellschaft? Wo wir stehen und wo wir hin müssen. erwachsenenbildung.at, 30, 1-11.

World Bank (2014). STEP Skills Measurement. Snapshot 2014. www.worldbank.org/content/dam/ Worldbank/Feature\%20Story/Education/STEP\%20Snapshot\%202014_Revised_June\%2020\%2020 14\%20(final).pdf. Zugegriffen: 24. Sept. 2019. Unter Mitarbeit von A. Valerio, M. L. Sanchez Puerta, P. Gäelle, T. Rajadel \& S. Monroy Taborda. World Bank.

Yasukawa, K. (2018). The workplace as a site for learning critical numeracy practice. In K. Yasukawa, A. Rogers, K. Jackson \& B. V. Street (Hrsg.), Numeracy as social practice. Global and local perspectives (S. 225-240). Milton: Routledge.

Yasukawa, K., Rogers, A., Jackson, K., \& Street, B. V. (Hrsg.). (2018). Numeracy as social practice. Global and local perspectives. Milton: Routledge.

Zabal, A., Martin, S., Klaukien, A., Rammstedt, B., Baumert, J., \& Klieme, E. (2013). Grundlegende Kompetenzen der erwachsenen Bevölkerung in Deutschland im internationalen Vergleich. In B. Rammstedt (Hrsg.), Grundlegende Kompetenzen Erwachsener im internationalen Vergleich. Ergebnisse von PIAAC 2012 (S. 31-59). Münster: Waxmann.

Zeuner, C.; Pabst, A. \& Benz-Gydat, M. (im Druck) "I have to do something for my body and also for my mind": Numeracy practices as a startegy against vulnerability in old age. First results of an explorative study. ZDM Mathematics Education, 52(2).

Publisher's Note Springer Nature remains neutral with regard to jurisdictional claims in published maps and institutional affiliations. 